\title{
Considerations of temperature stability in a cylindrical plasma
}

S. Neil Rasband

judith@conselle.com

Yao-Hui Xu

Follow this and additional works at: https://scholarsarchive.byu.edu/facpub

Part of the Astrophysics and Astronomy Commons, and the Physics Commons

\section{Original Publication Citation}

Rasband, S. N. and Yao H. Xu."Considerations of temperature stability in a cylindrical plasma."

Physics of Fluids 29 (1986): 2639-2647.

\section{BYU ScholarsArchive Citation}

Rasband, S. Neil and Xu, Yao-Hui, "Considerations of temperature stability in a cylindrical plasma" (1986). Faculty Publications. 752.

https://scholarsarchive.byu.edu/facpub/752 


\title{
Considerations of temperature stability in a cylindrical plasma
}

\author{
S. N. Rasband and Yao-Hui Xu \\ Department of Physics and Astronomy, Brigham Young University, Provo, Utah 84602
}

(Received 12 August 1985; accepted 7 May 1986)

\begin{abstract}
A variational formulation is used to consider thermal equilibrium and stability for a cylindrical plasma modeled by dissipative magnetohydrodynamics. The parameter dependence of equilibrium solutions and their stability is followed using homotopy techniques. Solution branches and their connections are studied in relation to the applied fields.
\end{abstract}

\section{INTRODUCTION}

Recently a variational principle has been developed for treating plasma problems modeled by dissipative magnetohydrodynamics (DMHD). ${ }^{1}$ This variational method is based on the work of Prigogine et al. in nonequilibrium thermodynamics. ${ }^{2-9}$ Coupled with finite element methods and cubic $B$ splines, this variational technique becomes particularly effective for finding stationary solutions to problems in DMHD. In this article we make extensive use of this technique to study the thermal equilibrium and stability properties of a thermally conducting, Ohmically heated plasma that is modeled by DMHD. The study is carried out in cylindrical geometry with the axial magnetic field a constant and typically much stronger than the poloidal field, representing an approximation to the fields of a tokamak.

In Sec. II we review briefly the local potential (LP) formulation and its physical interpretation. We also obtain appropriate LP functionals for the physical model of interest. In Sec. III we do the necessary scaling to obtain dimensionless variables and specialize to cylindrical coordinates. Section IV contains a discussion of the thermal stability problem for a cylindrical model of a tokamak and the dependence of equilibrium on boundary conditions and other physical parameters. In Sec. V we describe the results of our study employing the analysis of Sec. IV. We also discuss the accuracy of our numerical methods. Section VI contains our conclusions.

\section{MINIMIZATION OF THE LOCAL POTENTIAL}

\section{A. Physical interpretation and the variations}

For brevity we let $\phi(x, t)$ denote a composite field variable of position $\mathrm{x}$ and time $t$. The components of $\phi(\mathbf{x}, t)$ may consist of temperature $T(\mathbf{x}, t)$, magnetic field $B(x, t)$, velocity $\mathbf{v}(\mathbf{x}, t)$, etc., i.e., $\phi(\mathbf{x}, t)=[T(\mathbf{x}, t), \mathbf{B}(\mathbf{x}, t), \mathbf{v}(\mathbf{x}, t), \ldots]$. The LP functional $\Phi\left(\phi(x, t), \phi_{0}(x, t)\right)$ is a volume integral over terms involving components of two composite fields $\phi(x, t)$ and $\phi_{0}(x, t)$. The field $\phi_{0}(x, t)$, with the zero subscript, is interpreted as the average value of the fields at the position $\mathbf{x}$ and the time $t$. The field $\phi(x, t)$, without the subscript, is viewed as including fluctuations of the fields away from the average values. The minimization of $\Phi\left(\phi, \phi_{0}\right)$ with respect to $\phi$ by the methods of variational calculus, is interpreted as calculating the most probable value $\phi_{*}(\mathbf{x}, t)$ given $\phi_{0}(x, t)$. Here $\Phi\left(\phi_{*}, \phi_{0}\right)$ is interpreted as a functional minimum local to $\phi_{0}$. A posteriori we apply the "subsidiary condition"2 that $\phi_{*}(x, t)=\phi_{0}(x, t)$. This subsidiary condition is to be interpreted as demanding that the most probable state $\phi_{*}(x, t)$, obtained from the minimization procedure, coincide with the average state $\phi_{0}(x, t)$. The partial differential equations (pde) that result from this procedure are those which determine the state of the system. It is, in fact, our foreknowledge of these pde that guides us in choosing an acceptable $\Phi\left(\phi, \phi_{0}\right)$.

The choice of $\Phi\left(\phi, \phi_{0}\right)$ is further restricted by the demand that $\Phi\left(\phi, \phi_{0}\right)>\Phi\left(\phi_{0}, \phi_{0}\right)$ for all fluctuations $\phi(\mathbf{x}, t)$ away from $\phi_{0}(x, t)$. In other words, $\Phi\left(\phi_{*}, \phi_{0}\right)$ must be a true minimum of $\Phi\left(\phi, \phi_{0}\right)$ for all $\phi(x, t)$.

Within these constraints a considerable number of LP functionals are still possible. We further choose among the possibilities by insuring that the proper boundary conditions are incorporated. Lastly, we consider the integrals that must be computed numerically in our approximation scheme and choose a LP functional that is computationally economical.

\section{B. Local potentials for $\pi(x, t)$ and $B(x, t)$}

For simplicity we restrict ourselves in this work to a consideration of DMHD where the number density $n$ is assumed constant in $\mathbf{x}$ and $t$. We further assume the velocity field to be identically zero and simply ignore the momentum balance equation. For realistic solutions one certainly must demand force balance, and stability as well, but we leave this additional complexity to a subsequent study. We are interested presently in the question of thermal stability and the dependence of stability on the parameters of the system. There is no difficulty in principle in treating more general situations with a LP functional.

With the previous stated assumptions the familiar pde for local energy balance and the magnetic field are

$$
\begin{aligned}
& \frac{n k}{\gamma-1} \frac{\partial T}{\partial t}=\nabla \cdot(\kappa \nabla T)+\eta J^{2}-S, \\
& \frac{1}{c} \frac{\partial \mathbf{B}}{\partial t}=-\nabla \times \eta \mathbf{J},
\end{aligned}
$$

respectively. The quantities of interest in Eqs. (1) and (2) are, in addition to Boltzmann's constant $k$ and velocity of light $c: T(\mathbf{x}, t)$ : temperature distribution, $\mathbf{B}(\mathbf{x}, t)$ : magnetic field, $\mathbf{J}(\mathbf{x}, t)=(c / 4 \pi) \nabla \times \mathbf{B}$ : current density, $S(\mathbf{x}, t)$ : sinks (sources) of energy, $\eta(T)$ : resistivity, and $\kappa(T, \mathrm{~B})$ : thermal conductivity.

We illustrate the procedure for obtaining a LP by considering Eq. (1) in detail. Further details for Eq. (2) are given in Ref. 1. 
In obtaining a LP for Eq. (1) it is necessary to consider a variation of $T$, however, $(1 / T)$ turns out to be more convenient. Consider the first term on the right-hand side of Eq. (1). Then $\nabla \cdot(\kappa \nabla T)=-\nabla \cdot\left[\kappa T^{2} \nabla(1 / T)\right]$. Multiplying by $\delta(1 / T)$, integrating over the volume $V$, and performing an integration by parts results in

$$
\begin{aligned}
\int_{V} d v \delta\left(\frac{1}{T}\right) \nabla \cdot(\kappa \nabla T)= & -\int_{V} d v \nabla \cdot\left[\delta\left(\frac{1}{T}\right) \kappa T^{2} \nabla\left(\frac{1}{T}\right)\right] \\
& +\int_{V} d v \kappa T^{2} \nabla\left(\frac{1}{T}\right) \cdot \nabla\left[\delta\left(\frac{1}{T}\right)\right]
\end{aligned}
$$

A variation as described in Sec. II A shows that an appropriate quadratic term for for the LP functional is

$$
\int_{V} d v \frac{\kappa_{0} T_{0}^{2}}{2}\left|\nabla\left(\frac{1}{T}\right)\right|^{2}
$$

The zero subscripts are placed on $\kappa$ and $T^{2}$ to prevent them from being varied; then only $|\nabla(1 / T)|^{2}$ is varied with $\delta(1 /$ $T$ ). The other terms for Eq. (1) are then easily added in the integral by placing zero subscripts on them and giving them a multiplying factor of $(1 / T)$. Thus an appropriate LP for Eq. (1) is

$$
\begin{aligned}
\Phi_{T}= & \int_{V} d v\left[\frac{\kappa_{0} T_{0}^{2}}{2}\left|\nabla\left(\frac{1}{T}\right)\right|^{2}\right. \\
& \left.+\frac{1}{T}\left(\eta_{0} J_{0}^{2}-S_{0}-\frac{n k}{\gamma-1} \frac{\partial T_{0}}{\partial t}\right)\right] .
\end{aligned}
$$

It is straightforward to check that the variational procedure outlined in Sec. II A applied to Eq. (4) returns Eq. (1). In a similar fashion one can readily check that

$$
\Phi_{B}=\int_{V} d v\left(\frac{\eta_{\sigma^{J}}}{2}+\frac{1}{4 \pi} \mathbf{B} \cdot \frac{\partial \mathbf{B}_{0}}{\partial t}\right)
$$

is a suitable LP for Eq. (2).

The LP functionals are not unique and indeed a single LP functional for both fields can be given as

$$
\begin{aligned}
\Phi= & \int_{V} d v\left\{\frac{\kappa_{0}}{2} T_{0}^{2}\left|\nabla\left(\frac{1}{T}\right)\right|^{2}+\frac{\eta_{0}}{T} J^{2}-\frac{1}{T} \frac{\partial}{\partial t}\left(\frac{n k T_{0}}{\gamma-1}\right)\right. \\
& \left.-\frac{1}{T} S_{0}+\frac{c}{2 \pi} \mathbf{B} \cdot\left[\frac{1}{T_{0} c} \frac{\partial \mathbf{B}_{0}}{\partial t}-\nabla\left(\frac{1}{T_{0}}\right) \times \eta_{0} \mathbf{J}_{0}\right]\right\},
\end{aligned}
$$

but we will make no use of this LP because it complicates integrals in the B-field equation with factors of $(1 / T)$.

The variation of Eqs. (4) and (5) also imply the boundary conditions

$$
\begin{aligned}
& \left.\delta(1 / T) \kappa \nabla T \cdot \hat{\mathbf{n}}\right|_{\partial \mathrm{V}}=0, \\
& \left.(c \eta / 4 \pi)(\delta \mathbf{B} \times \mathbf{J}) \cdot \hat{\mathbf{n}}\right|_{\partial V}=0,
\end{aligned}
$$

respectively. Here $\partial V$ denotes the bounding surface of $V$ and $\hat{\mathbf{n}}$ the outward pointing normal vector.

For our purposes $T$ will always be fixed on the boundary and hence $\delta(1 / T)$ vanishes there. Consequently in this paper, the LP functional Eq. (4) and the associated boundary condition Eq. (7) will always be appropriate for the temperature.

In contrast, boundary conditions satisfying Eq. (8) are not always convenient or suitable for the magnetic field $B$. If this is the case, a LP different from Eq. (5) is necessary. We postpone the detailed consideration of these additional situations until we specialize to cylindrical geometry.

\section{SPECIALIZATION TO CYLINDAICAL COORDINATES AND SCALED VARIABLES}

\section{A. Cylindrical symmetry}

We envision the plasma in a long cylindrical container, symmetric about the axis of this container. Consequently, we make the simplifying assumption that the only spatial dependence for any field quantity is in the radial direction. Then

$$
\int_{V} d v \rightarrow \int_{0}^{a} r d r, \quad \boldsymbol{\nabla} f \rightarrow \frac{\partial f}{\partial r} \hat{\mathbf{r}}, \quad \boldsymbol{\nabla} \cdot \mathbf{A} \rightarrow \frac{1}{r} \frac{\partial}{\partial r}\left(r A_{r}\right),
$$

where $f$ is a scalar function and $\boldsymbol{A}$ is a vector function. The quantity $a$ is the assumed radius of the cylindrical chamber containing the plasma.

We further assume that $\mathbf{B}(\mathbf{x}, t)=\left[0, B_{\theta}(r, t), B_{z}\right]$, where $B_{z}$ is constant. Similarly $T(\mathbf{x}, t)=T(r, t)$.

\section{B. Scaling}

All quantities are scaled relative to values denoted as follows: $T_{s}$ : scale temperature, nominally taken to be $1 \mathrm{keV}$; $B_{s}$ : scale magnetic field, nominally taken to be $1 \mathrm{kG} ; a_{s}$ : scale length, nominally taken to be $20 \mathrm{~cm}$; and $n_{s}$ : scale density, nominally taken to be $10^{14} \mathrm{~cm}^{-3}$.

For the transport coefficients we take

$$
\begin{aligned}
\kappa & =4.37 \times 10^{2} \frac{n^{2}}{B^{2} T^{n_{c}}} \frac{\operatorname{erg}}{\operatorname{deg~cm~sec}} \frac{\left(n_{s} \mathrm{~cm}^{3} / 10^{14}\right)^{2}}{\left(B_{s} / \mathrm{kG}\right)^{2}\left(T_{s} / \mathrm{keV}\right)^{n_{c}}} \\
& \equiv \frac{\bar{\kappa} n^{2}}{B^{2} T^{n_{c}}},
\end{aligned}
$$

$\eta=3.64 \times 10^{-18} \frac{1}{T^{n_{r}}} \sec \left(\frac{T_{s}}{\mathrm{keV}}\right)^{-n_{r}} \equiv \frac{\bar{\eta}}{T^{n_{r}}}$.

With the exponents $n_{c}$ and $n_{r}$ equal to $\frac{1}{2}$ and $\frac{3}{2}$, respectively, these transport coefficients represent classical values. We note that $B^{2}=B_{\theta}^{2}+B_{z}^{2}$ with constant $B_{z}$.

We use the resistive diffusion time $\tau_{r} \equiv 4 \pi a_{s}^{2} / \bar{\eta} c^{2}$ to scale the time variable and write $\tau=t / \tau_{r}$.

The only loss term $S$ that we consider here is Bremsstrahlung loss. Other heating or loss terms can be included in a similar manner:

$$
S=5.35 \times 10^{4} \frac{\mathrm{erg}}{\mathrm{cm}^{3} \mathrm{sec}} n^{2} T^{n_{B}}\left(\frac{n_{s} \mathrm{~cm}^{3}}{10^{14}}\right)^{2}\left(\frac{T_{s}}{\mathrm{keV}}\right)^{n_{B}},
$$

where $n_{B}=\frac{1}{2}$.

We also define $x_{w}=a / a_{s}$, with $x=r / a_{s}$. Then with these assumptions concerning geometry and scaling it is straightforward to obtain specific forms for the LP in Eqs. (4) and (5),

$$
\begin{aligned}
\Phi_{T}= & \text { const } \int_{0}^{x_{w}} x d x\left\{\frac{T_{0}^{\left(2-n_{c}\right)}}{2 B_{0}^{2}}\left[\frac{\partial}{\partial x}\left(\frac{1}{T}\right)\right]^{2}\right. \\
& \left.+\frac{1}{T}\left[\frac{\mu}{T_{0}^{n_{r}}}\left(\frac{\partial B_{\theta}}{\partial x}+\frac{B_{\theta}}{x}\right)^{2}-\beta T_{0}^{n_{B}}-v \frac{\partial T_{0}}{\partial \tau}\right]\right\},
\end{aligned}
$$


$\Phi_{B}=$ const $\int_{0}^{x_{w}} x d x\left[\frac{1}{2 T_{0}^{n_{r}}}\left(\frac{\partial B_{\theta}}{\partial x}+\frac{B_{\theta}}{x}\right)^{2}+B_{\theta} \frac{\partial B_{0 \theta}}{\partial \tau}\right]$.

The dimensionless constants occurring in Eq. (12) are

$\mu=4.12 \times 10^{-3}\left(\frac{B_{s}}{\mathrm{kG}}\right)^{4}\left(\frac{T_{s}}{\mathrm{keV}}\right)^{\left(n_{c}+n_{r}-1\right)}\left(\frac{n_{s} \mathrm{~cm}^{3}}{10^{14}}\right)^{-2}$,

$\beta=4.24 \times 10^{-3}\left(\frac{B_{s}}{\mathrm{kG}}\right)^{2}\left(\frac{a_{s}}{20 \mathrm{~cm}}\right)^{2}\left(\frac{T_{s}}{\mathrm{keV}}\right)^{\left(n_{B}+n_{c}-1\right)}$,

$v=1.25 \times 10^{-2}\left(\frac{B_{s}}{\mathrm{kG}}\right)^{2}\left(\frac{T_{s}}{\mathrm{keV}}\right)^{\left(n_{c}+n_{B}\right)}\left(\frac{n_{s} \mathrm{~cm}^{3}}{10^{14}}\right)^{-1}$.

One final bit of scaling remains. That is to change the scale of the independent variable $x$. The interval from 0 to $x_{w}$ will be subdivided into $N$ finite elements of equal length. Since it is convenient to compute cubic $B$ splines over finite elements, each of unit length, we change to a new radial variable given by the formula

$$
\xi=x N / x_{w} .
$$

With this change of scale Eqs. (12) and (13) become

$$
\begin{aligned}
\Phi_{T}= & \text { const } \int_{0}^{N} \xi d \xi\left\{\frac{T_{0}^{2-n_{c}}}{2 B_{0}^{2}}\left[\left(\frac{1}{T}\right)\right]^{2}+\frac{1}{T}\right. \\
& \left.\times\left[\frac{\mu}{T_{0}^{n_{r}}}\left(B_{\theta}^{\prime}+\frac{B_{\theta}}{\xi}\right)^{2}-\frac{\beta x_{w}^{2}}{N^{2}} T_{0}^{n_{B}}-\frac{v x_{w}^{2}}{N^{2}} \frac{\partial T_{0}}{\partial \tau}\right]\right\}, \\
\Phi_{B}= & \text { const } \int_{0}^{N} \xi d \xi\left[\frac{1}{2 T_{0}^{n_{r}}}\left(B_{\theta}^{\prime}+\frac{B_{\theta}}{\xi}\right)^{2}+B_{\theta} \frac{x_{w}^{2}}{N^{2}} \frac{\partial B_{0 \theta}}{\partial \tau}\right],
\end{aligned}
$$

where primes denote differentiation with respect to $\xi$.

Since we are interested in stationary solutions with no time dependence, the terms in Eqs. (15) and (16) involving derivatives with respect to $\tau$ are dropped. We also drop the multiplicative constants in front of the integrals since they can be eliminated by a simple rescaling of the LP,

$$
\begin{gathered}
\Phi_{T}=\int_{0}^{N} \xi d \xi\left\{\frac{T_{0}^{2-n_{c}}}{2 B_{0}^{2}}\left[\left(\frac{1}{T}\right)\right]^{2}+\frac{1}{T}\right. \\
\left.\times\left[\frac{\mu}{T_{0}^{n_{r}}}\left(B_{0 \theta}^{\prime}+\frac{B_{0 \theta}}{\xi}\right)^{2}-\frac{\beta x_{w}^{2}}{N^{2}} T_{0}^{n_{B}}\right]\right\}, \\
\Phi_{B}=\int_{0}^{N} \xi d \xi \frac{1}{2 T_{0}^{n_{r}}}\left(B_{\theta}^{\prime}+\frac{B_{\theta}}{\xi}\right)^{2} .
\end{gathered}
$$

For the stationary case the pde for $B_{\theta}$ resulting from Eq. (18) can be integrated once to give

$$
\frac{1}{T^{n_{r}}}\left(B_{\theta}^{\prime}+\frac{B_{\theta}}{\xi}\right)=\frac{x_{w}}{N} E,
$$

where $E$ (a constant of integration) is the scaled electric field in the axial direction. Note that the left-hand side of Eq. (19) is a scaled form of $\eta J_{z}$, which is $E_{z}$ from Ohm's law. To obtain conventional units $(\mathrm{V} / \mathrm{m})$ from values of the dimensionless variable $E$, multiply by the factor 0.43 .

If we use the LP of Eq. (18), which demands as a boundary condition that $B_{\theta}(N)=$ const, Eq. (19) must still be satisfied. In fact the required constancy of the left-hand side of Eq. (19) serves as a useful check on the accuracy of the numerical solution and gives an indication of the sufficiency of the number of finite elements $N$.

We can, however, make even more direct use of Eq. (19). We can take advantage of the fact that one integration has been performed with Eq. (19). We can write a LP for Eq. (19) directly. If we do this then $E$ becomes a parameter of the solution instead of $B_{\theta}(N)=B_{w}$. Either $E$ or $B_{w}$ can be specified, providing we use the appropriate LP. Specifying $B_{w}$ is equivalent to specifying the total axial current, as is easily shown by Ampère's law.

Following the same procedure as outlined in Sec. II B, we obtain a LP for Eq. (19),

$$
\bar{\Phi}_{B}=\int_{0}^{N} \xi d \xi\left[\frac{B_{\theta}^{2}}{2 \xi}+B_{\theta}\left(B_{0 \theta}^{\prime}-\frac{x_{w}}{N} E T_{0}^{n_{r}}\right)\right] .
$$

It is straightforward to check that $\bar{\Phi}_{B}$ under a variation as described in Sec. II A gives back Eq. (19) without implying any boundary conditions.

Thus if we compute equilibria where the total axial current, i.e., $B_{w}$ is specified, we use the LP in Eq. (18). If we instead wish to specify the axial electric field $E$, then we use the LP in Eq. (20).

\section{EQUILIBRIUM AND STABILITY}

\section{A. Spllne expansions}

As mentioned in the previous section we partition the radial interval into $N$ finite elements each of unit length. The chamber wall where $r=a$ then corresponds to $\xi=N$ and the knot points occur at the integer values $(0,1,2, \ldots, N)$. We wish to expand $T(\xi)$ and $B_{\theta}(\xi)$ in terms of splines defined on the finite elements in the usual way. However, before one can specify the spline basis functions it is necessary to specify boundary conditions.

The boundary condition on $T(\xi)$ is that

$$
T^{\prime}(0)=0, \quad T(N)=T_{w}=\text { const. }
$$

Using the summation convention for repeated indices we write

$$
1 / T(\xi)=T^{i} \phi_{i}(\xi)+1 / T_{w}, \quad i=1, \ldots, N+1,
$$

where $T_{w}$ is the fixed value of the temperature at the wall. The $\phi_{i}(\xi)$ are spline basis functions given in terms of cubic $B$ splines in Table I. These spline basis functions satisfy $\phi_{i}^{\prime}(0)=0$ and $\phi_{i}(N)=0$ for all $i$. There are exactly $N+1$ knot coefficients to be determined.

The boundary condition on $B_{\theta}(\xi)$ implied by the LP of Eq. (18) is that

$$
B_{\theta}(0)=0, \quad B_{\theta}(N)=B_{w}=\text { const. }
$$

Again using the summation convention, we expand $B_{\theta}$ in terms of spline basis functions,

$$
B_{\theta}(\xi)=B \hat{i}_{i}(\xi), \quad \hat{i}=1, \ldots, N+2,
$$

where $B^{N+2}=6 B_{w}$ for the spline normalization chosen. Again the basis functions $\bar{\psi}_{i}$ are given in terms of cubic $B$ splines and the appropriate combinations to satisfy the boundary conditions are tabulated in Table I. There are exactly $N+1$ knot coefficients $B^{\hat{i}}$ to be determined.

For the LP in Eq. (20), which is appropriate when we 
TABLE I. Spline basis functions as used in the text for expanding field variables. The expansion functions are numbered consecutively beginning at 1 to $N+2$ for indices with carets. Other indices run from 1 to $N+1$. The labels on the $B$ splines denote the knot at which they have their maximum

\begin{tabular}{ll}
\hline \hline Basis function set & $\begin{array}{l}B \text { spline } \text { combinations } \\
\text { comprising the basis function set }\end{array}$ \\
\hline$\left\{\phi_{i}\right\}=\left\{B_{0}, B_{1}+B_{-1}, B_{2}, \ldots, B_{N-2}, B_{N-1}-B_{N+1}, B_{N}-4 B_{N+1}\right\}$ \\
$\left\{\bar{\psi}_{i}\right\}=\left\{B_{0}-4 B_{-1}, B_{1}-B_{-1}, B_{2}, \ldots, B_{N-2}, B_{N-1}-B_{N+1}, B_{N}-4 B_{N+1}, B_{N+1}\right\}$ \\
$\left\{\psi_{i}\right\}=\left\{B_{0}-4 B_{-1}, B_{1}-B_{-1}, B_{2}, \ldots, B_{N+1}\right\}$ \\
$\left\{\tilde{\psi}_{i}\right\}=\left\{B_{0}-4 B_{-1}, B_{1}-B_{-1}, B_{2}, \ldots, B_{N-2}, B_{N-1}+\frac{(3 N-1)}{(3 N+1)} B_{N+1}, B_{N}-\frac{4}{(3 N+1)} B_{N+1}\right\}$
\end{tabular}

${ }^{a} B$ splines are normalized to have unit area under their curves.

wish to specify $E$ for the equilibrium solution, a different set of spline basis functions must be used. We still make an expansion of the form

$$
B_{\theta}(\xi)=B^{\hat{i}} \psi_{i}(\xi), \quad \hat{i}=1, \ldots, N+2 .
$$

But now the basis functions $\psi_{i}(\xi)$ must only satisfy the one boundary condition $\psi_{i}(0)=0$. The combinations are also listed in Table I. In this case there are $N+2$ knot coefficients $B^{\hat{i}}$ to be determined.

To obtain the stationary solutions either LP of Eq. (18) or Eq. (20) can be used. We have used both and checked the solutions one against the other. There seems to be no advantage to one scheme over the other and the solution set obtained is the same. Consequently, we henceforth consider only equilibria that use $\bar{\Phi}_{B}$ as the LP.

\section{B. Equillbrium equations}

With the spline expansion of the fields of interest we can now perform the variation as outlined in Sec. II A. We compute $\partial \Phi_{T} / \partial T^{i}$ using Eq. (17), where $T(\xi)$ is given in terms of the knot coefficients $T^{i}$ and $T_{0}(\xi)$ in terms of the knot coefficients $T_{0}^{i}$. After having $\partial \Phi_{T} / \partial T^{i}$, then to implement the subsidiary condition all $T^{i}$ are set equal to $T_{0}^{i}$. Similarly $B_{\theta}$ and $B_{0 \theta}$ correspond to the knot coefficients $B^{\hat{i}}$ and $B_{0}^{\hat{i}}$, respectively. In analogous fashion we find $\partial \bar{\Phi}_{B} / \partial B^{\hat{i}}$ and then set all $B^{\hat{i}}=B_{0}^{\hat{i}}$. The result is a system of nonlinear equations for the $T_{0}^{i}$ and $B_{0}^{\hat{i}}$, the solution of which gives a spline approximation to a stationary solution of the pde.

These nonlinear equations are of the form

$$
\begin{aligned}
& U_{i j} T_{0}^{j}+V_{i}=0, \\
& W_{i j} B_{0}^{\hat{j}}-\left(x_{w} E / N\right) X_{\hat{i}}=0 .
\end{aligned}
$$

The indices without carets over them range from 1 to $N+1$, the others from 1 to $N+2$. The matrices $U_{i j}, V_{i}, W_{\hat{i} j}, X_{\hat{i}}$ occurring in Eqs. (26) and (27) are given in the Appendix. We note here only that Eqs. (26) and (27) are highly nonlinear since the matrices depend on the knot coefficients $T_{0}^{i}$ and $B_{0}^{\hat{i}}$, with the exception of $W_{\hat{i}}$. The matrix $X_{\hat{i}}$ also does not depend on the $B_{0}^{\hat{j}}$.

The standard Newton method is an effective technique for solving Eqs. (26) and (27) for the $T_{0}^{j}$ and the $B_{0}^{j}$ if a good initial guess is available. To obtain such starting values we have used an initial value in tegrator for ordinary differential equations (ode). We note that the equilibrium equations for $T$ and $B_{\theta}$ in the scaled variable $x=r / a_{s}$ are

$$
\begin{aligned}
& \frac{d}{d x}\left(\frac{1}{B^{2} T^{n_{c}}} \frac{d T}{d x}\right)+\mu E^{2} T^{n_{r}}-\beta T^{n_{B}}=0, \\
& \frac{1}{T^{n_{r}}}\left(\frac{d B_{\theta}}{d x}+\frac{B_{\theta}}{x}\right)-E=0 .
\end{aligned}
$$

Defining $\quad y_{1}(x)=\left(1 / B^{2} T^{n_{c}}\right)(d T / d x), \quad y_{2}(x)=T(x)$, $y_{3}(x)=B_{\theta}(x)$, then Eqs. (28) and (29) become a system of three ode. We note that $y_{1}(0)=0$ and $y_{3}(0)=0$. Consequently,. we can specify a value $y_{2}(0)=T_{c}$ (central temperature) and integrate forward in $x$ until $T$ takes on the value $T_{w}$ at some $x_{0}$, which we then identify with $x_{w}$. This does not happen for all values of $T_{c}$ of course, but when it does the functions $T(x)$ and $B_{\theta}(x)$ are a solution to Eqs. (28) and (29) with $T=T_{w}$ at $x=x_{w}$ and a given value of $E$. Solving Eqs. (28) and (29) in this way gives good starting values for solving Eqs. (26) and (27). We also can use this procedure to check subsequent solutions of Eqs. (26) and (27). Solving the Eqs. (28) and (29) using an initial-value ode solver gives solutions of high accuracy but is computationally expensive for studying the parameter dependence of solutions.

To study the dependence of solutions on parameters such as $x_{w}$ (size of the containment vessel) and $E$ (axial electric field) we use a homotopy routine. ${ }^{10-12}$ These homotopy routines allow the computation of a continuous curve of solutions and permit the display of solution dependence on any parameter of choice. We have focused in this article on the dependence of solutions on parameters that can be changed by the experimenter, such as $E, B_{w}$, and $x_{w}$, as opposed to the value of transport coefficients and exponents of temperature in these transport coefficients.

\section{Stability}

To study the stability of the equilibrium solutions found by the methods described in the previous subsection we let $T(\xi, \tau)=T_{0}(\xi)+t(\xi, \tau) \quad$ and $\quad B_{\theta}(\xi, \tau)=B_{0 \theta}(\xi)$ $+b_{\theta}(\xi, \tau)$, where $T_{0}$ and $B_{0 \theta}$ are the presumed known equilibrium solutions. We consider perturbations from the equilibrium solutions to compute the linearized operator associated with the pde of Eqs. (1) and (2). We scale as before and find after a tedious but straightforward computation that 


$$
\begin{aligned}
\frac{v x_{w}^{2}}{N^{2}} & \frac{\partial t}{\partial \tau} \\
= & \frac{1}{\xi} \frac{\partial}{\partial \xi}\left[\xi\left(\frac{t^{\prime}}{B_{0}^{2} T_{0}^{n_{c}}}-\frac{2 B_{0 \theta} T_{0}^{\prime} b_{\theta}}{\left(B_{0}^{2}\right)^{2} T_{0}^{n_{c}}}-\frac{n_{c} T_{0}^{\prime} t}{B_{0}^{2} T_{0}^{n_{c}+1}}\right)\right] \\
& -t\left(\frac{\beta x_{w}^{2} n_{B}}{N^{2}} T_{0}^{n_{B}-1}+\frac{\mu n_{r} x_{w}^{2} E^{2}}{N^{2}} T_{0}^{n_{r}-1}\right) \\
& +\frac{2 \mu x_{w} E}{N}\left(b_{\theta}^{\prime}+\frac{b_{\theta}}{\xi}\right) \\
\frac{x_{w}^{2}}{N^{2}} \frac{\partial b_{\theta}}{\partial \tau} & =\frac{\partial}{\partial \xi}\left[\frac{1}{T_{0}^{n_{r}}}\left(b_{\theta}^{\prime}+\frac{b_{\theta}}{\xi}\right)-\frac{n_{r} x_{w} E}{N} \frac{t}{T_{0}}\right]
\end{aligned}
$$

The boundary conditions placed on the perturbed fields are

$$
\begin{aligned}
& t^{\prime}(0)=0, \quad t(N)=0, \\
& b_{\theta}(0)=0,\left.\quad\left(b_{\theta}^{\prime}+b_{\theta} / \xi\right)\right|_{N}=0 .
\end{aligned}
$$

Equations (30) and (31) along with the boundary conditions in Eqs. (32) and (33) can also be formulated in terms of a LP. Following the outline previously given for constructing such functionals, we obtain

$$
\begin{aligned}
\Psi_{t}= & \int_{0}^{N} \xi d \xi\left\{\frac{-\left(t^{\prime}\right)^{2}}{2 B_{0}^{2} T_{0}^{n_{c}}}+\frac{t^{\prime} T_{0}^{\prime}}{B_{0}^{2} T_{0}^{n_{c}}}\left(\frac{2 B_{0 \theta} b_{0 \theta}}{B_{0}^{2}}+\frac{n_{c} t_{0}}{T_{0}}\right)\right. \\
& +t\left[\frac{-v x_{w}^{2}}{N^{2}} \frac{\partial t_{0}}{\partial \tau}-\left(\frac{\beta x_{w}^{2} n_{B} T_{0}^{\left(n_{B}-1\right)}}{N^{2}}\right.\right. \\
& \left.\left.\left.+\frac{\mu n_{r} x_{w}^{2} E^{2} T_{0}^{\left(n_{r}-1\right)}}{N^{2}}\right) t_{0}+\frac{2 \mu x_{w} E}{N}\left(b_{0 \theta}^{\prime}+\frac{b_{0 \theta}}{\xi}\right)\right]\right\},
\end{aligned}
$$

$$
\begin{aligned}
\Psi_{b}= & \int_{0}^{N} \xi d \xi\left\{\frac{1}{2 T_{0}^{n_{r}}}\left(b_{\theta}^{\prime}+\frac{b_{\theta}}{\xi}\right)^{2}\right. \\
& \left.+b_{\theta}\left[\frac{n_{r} x_{w} E}{N}\left(\frac{t_{0}}{T_{0}}\right)^{\prime}+\frac{x_{w}^{2}}{N^{2}} \frac{\partial b_{0 \theta}}{\partial \tau}\right]\right\} .
\end{aligned}
$$

For the perturbation fields we use the expansions

$$
\begin{aligned}
& t(\xi, \tau)=t^{i}(\tau) \phi_{i}(\xi), \\
& b_{\theta}(\xi, \tau)=b^{i}(\tau) \tilde{\psi}_{i}(\xi) .
\end{aligned}
$$

The set $\left\{\phi_{i}(\xi)\right\}$ (which has been used previously) and the set $\left\{\tilde{\psi}_{i}\right\}$, which are appropriate for the boundary condition Eq. (33), are given in Table I.

Proceeding as in Sec. IV B gives the following linear matrix equations for $t_{0}^{i}$ and $b_{0}^{i}$ :

$$
\begin{aligned}
\frac{d t_{0}^{j}}{d \tau} & \\
= & \frac{N^{2}}{v x_{w}^{2}}\left(Y^{-1}\right)^{j i}\left[\left(A_{i k}-C_{i k}-\frac{\beta x_{w}^{2} n_{B}}{N^{2}} D_{i k}-\mu n_{r} E_{i k}\right)\right. \\
& \left.\times t_{0}^{k}+\left(B_{i k}+2 \mu F_{i k}\right) b_{0}^{k}\right] \\
\frac{d b_{0}^{j}}{d \tau}= & \frac{-N^{2}}{x_{w}^{2}}\left(Z^{-1}\right)^{j i}\left(\frac{n_{r} x_{w} E}{N} H_{i k} t_{0}^{k}+G_{i k} b_{0}^{k}\right)
\end{aligned}
$$

Once again the matrices $Y_{i j}, Z_{i j}, A_{i j}, B_{i j}, C_{i j}, D_{i j}, E_{i j}, F_{i j}, H_{i j}$, and $G_{i j}$ are given explicitly in the Appendix.

These matrix equations can be written symbolically in the form

$$
\left[\begin{array}{l}
\dot{t} \\
\dot{b}
\end{array}\right]=D\left[\begin{array}{l}
t \\
b
\end{array}\right]
$$

The eigenvalues of the matrix $D$ are an approximation to the first $2(N+1)(N$ is the number of finite elements) eigenvalues of the linear partial differential operator contained in Eqs. (30) and (31). In the following section we consider the accuracy of this approximation.

Eigenvalues of $D$ with positive real part indicate instability of the equilibrium solution about which the linearization has taken place. ${ }^{13}$

\section{EQUILIBRIUM AND STABILITY RESULTS}

All results reported here were obtained with a wall value for the temperature $T_{w}=0.01$. None of the conclusions or the general shapes of the curves to be displayed depend upon this choice. However, the actual magnitudes do. If $T_{w}$ is lowered to, say, 0.001 , everything else remaining the same, then the corresponding equilibrium solutions have lower values for the central temperature $T_{c}$ and the wall value of $B_{\theta}$. But, again there are no qualitative changes in the behavior of solutions or their stability. The value $T_{w}=0.01$ was chosen simply as a matter of convenience in obtaining equilibrium solutions. In a similar way we have arbitrarily cho$\operatorname{sen} B_{z}=2.0$.

The values of $B_{z}=2.0$ and $T_{w}=0.01$ were also partly chosen for comparison with the work in Refs. 14 and 15. The solution techniques employed in Ref. 14, although essentially numerical, were quite different than those in this work, and the techniques of Ref. 15 were analytical. It is therefore important to choose values for the parameters which clearly demonstrate the consistency of the present results with previous work.

This work is, in addition, complementary to that in Refs. 14 and 15 not only because of the different mathematical methods used, but because of the increased generality in the functional forms for the field dependencies of the transport coefficients. Although we have presented cases where $B_{z}>B_{\theta}$, and consequently the magnetic field dependence in $\kappa$ of Eq. (9) was slight, examples with $B_{\theta}$ and $B_{z}$ comparable are also easily computed. Indeed, all of the quantities $\bar{\kappa}, n_{c}$, $B_{z}, \bar{\eta}, n_{r}, n_{B}, \beta$ associated with dissipation or losses can be varied or made the parameter of a homotopy. Furthermore we have included Bremsstrahlung radiation as an example of losses, and elucidate in a following paragraph the dependence of a critical electric field on the functional dependence of these loss terms. We have also shown that with Bremsstrahlung losses alone, no bifurcations take place when one considers the total axial current, or $B_{w}$, versus $T_{c}$. Arbitrary heating or loss terms depending only on temperature and the magnetic field can be similarly studied.

The heating and loss terms play a critical role in determining the value of $E$ that divides the solutions into two classes: those with $T_{c}<T_{w}$ and those with $T_{c}>T_{w}$. The value of $E$ that separates the two classes we refer to as the critical $E$ and denote it $E_{c}$. We can calculate this $E_{c}$ from Eq. 
(28) as follows. We note that $-y_{1}(x)$ represents the heat current in the positive $x$ direction. For $T_{c}>T_{w}$ we expect this quantity to be positive and for $T_{c}<T_{w}$ we would expect it to be negative. The situation $y_{1}(x) \equiv 0$ would be for a constant temperature profile, where the temperature everywhere is just equal to the wall value $T_{w}$. We have demanded that $y_{1}(0)=0$ and therefore if $y_{1}(x)<0$, it must be the case that $\left[d y_{1}(x) / d x\right]<0$. Similarly, for $T_{c}<T_{w},\left[d y_{1}(x) /\right.$ $d x]>0$. Hence the critical value for $E$ occurs when $\left[d y_{1}(x) /\right.$ $d x]=0$, and this can occur only when the temperature everywhere equals $T_{w}$. Thus from Eq. (28) we find $\mu E_{c}^{2} T_{w}^{n_{r}}$ $-\beta T_{w}^{n_{B}}=0$, which gives

$E_{c}=\sqrt{(\beta / \mu)} T_{w}^{-\left(n_{r}-n_{B}\right) / 2}=1.01 T_{w}^{-\left(n_{r}-n_{B}\right) / 2}$.

For $T_{w}=0.01$ we have $E_{c}=10.1$, and if $T_{w}$ were chosen 0.001 then $E_{c}$ would be 31.9. It is thus clear that this critical value $E_{c}$ is determined by the heating and loss terms alone. For $E<E_{c}$ the homotopies of stable and unstable solutions are not connected through a limit point, i.e., a point of marginal stability. Although not focused on the critical values of the electric field, clear examples of this behavior in slab geometry are given in Ref. 15 . The manner in which the character of the homotopies changes as $E$ varies is considered in detail in the next section.

\section{A. Equilibria}

Equilibria do not exist for all choices of $x_{w}$ and $E$, and for situations where equilibria do exist, exactly two solutions exist for the same choice of parameters, one being stable and the other unstable. Figure 1 displays values of $x_{w}$ versus central temperature $T_{c}$ for two choices of $E$ above $E_{c}$. These curves represent a homotopy of equilibrium solutions where $x_{w}$ is the homotopy parameter. The peaks in these curves are limit points at which the equilibrium solutions change the character of their stability. Such a bifurcation is of the saddle-node type ${ }^{16}$ and because of the lack of special symmetry requirements on the system near the bifurcation point, is the

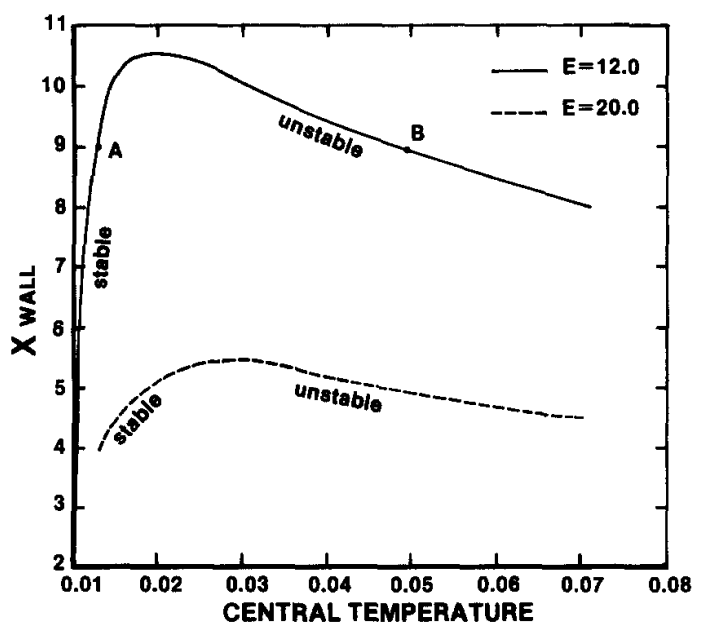

FIG. 1. For two fixed values of $E_{>} E_{c}$ a continuous homotopy of equilibrium solutions is plotted in the variables $x_{w}$ versus the central temperature. For a given value of $x_{w}$ either no equilibrium solution is possible or else two solutions exist, one stable and the other unstable. The peak of the curve is a limit point where the stability changes. Field profiles for the equilibria corresponding to points $\mathrm{A}$ and $\mathrm{B}$ are given in Fig. 2.

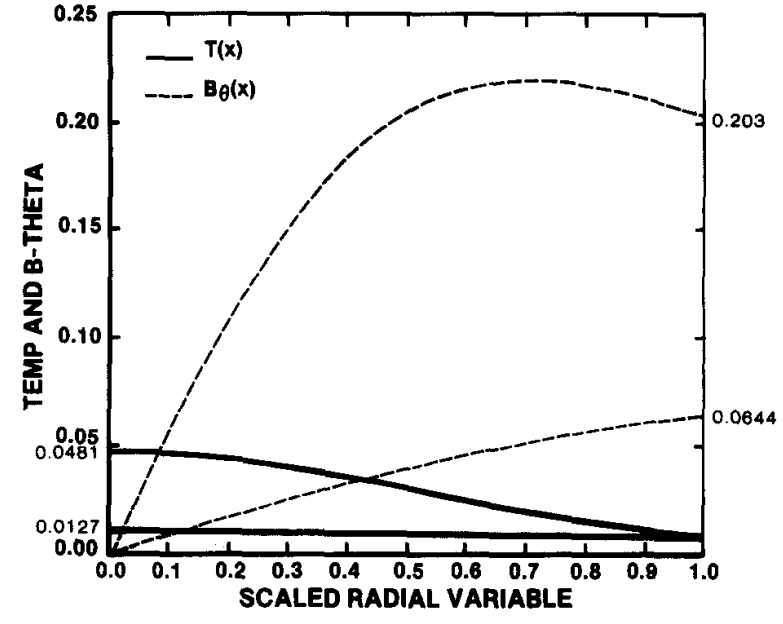

FIG. 2. Field profiles for the temperature $T$ and $B_{\theta}$. The profiles with the larger magnitudes correspond to point $B$ in Fig. 1 and smaller ones to point A.

type of bifurcation one would expect.

The basic reason for the lack of an equilibrium when $x_{w}$ or $E$ are too large is the inverse dependence of the resistivity on temperature. If the temperature at a given radius goes up, then the resistivity is smaller causing more current to flow at this radius, and consequently more Ohmic heating and a higher temperature leading to a runaway situation. It is obvious that a balance between heat sources and heat losses is what constitutes a stationary state, or equilibrium for the system. That there should be a maximum value of $x_{w}$ for which there are equilibrium solutions is physically reasonable. The wall is an important sink of energy. For the results presented in this study (a given choice of parameter values and loss terms) energy out through the wall is the most important loss. Therefore if the wall is too far away, all else remaining the same, then the gradients in the temperature are too small near the wall and too much heat is retained in the system, precluding the establishment of an equilibrium. As examples of stable and unstable solutions, the field profiles for the equilibrium solutions labeled by the points $A$ and

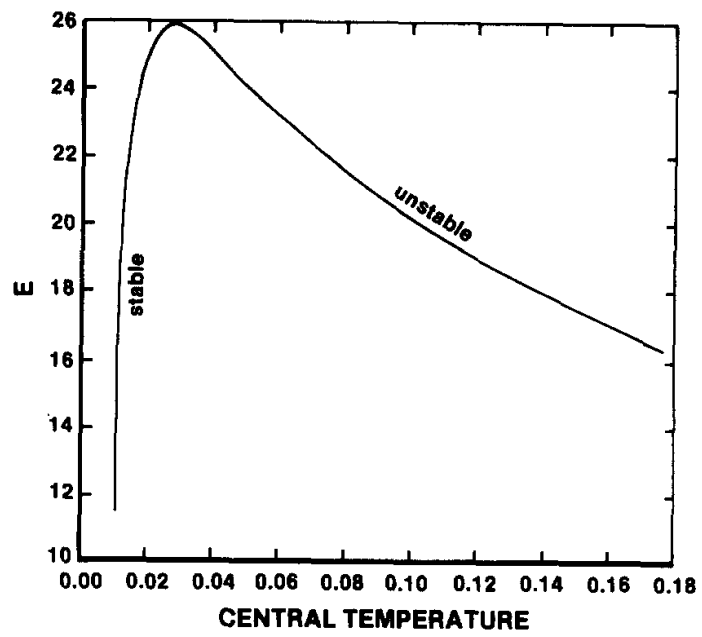

FIG. 3. A continuous homotopy of equilibria with $x_{w}=4.0$. The axial electric field $E$ is the homotopy parameter and is displayed against the central temperature $T_{c}$. 
B are given in Fig. 2. Reference 14 reports a study of the evolution of an equilibrium, such as that at point $B$, which evolves to a stable equilibrium.

Figure 3 is a similar homotopy curve of equilibria with a saddle-node bifurcation point. In this curve, however, $x_{w}=4.0$ is fixed and the scaled axial electric field $E$ is the homotopy parameter. The existence of a maximum $E$ for which there exist equilibrium solutions (stable or unstable) is a reflection of the fact that too much $E$ gives too much Ohmic heating for the heat to be conducted away and consequently a runaway situation again develops.

It is then interesting to examine the curve corresponding to the limit or bifurcation points as a function of $E$ and $x_{w}$. A portion of this curve is given in Fig. 4. We see that to the left of the curve two solutions are possible, one stable and one unstable. To the right of this curve no equilibrium solutions are possible. On the left the curve asymptotically approaches $E_{c}$.

The curve of Fig. 4 is obtained in the following way. We symbolically denote the nonlinear Eqs. (26) and (27) in the form

$$
F_{\alpha}\left(x^{\beta}\right)=0,
$$

where $x^{\beta}=\left(T_{0}^{i}, B_{0}^{\hat{i}}\right)$. A Greek index $(\alpha, \beta, \ldots)$ represents the union of the index sets for $i$ and $\hat{i}$. The Jacobian matrix $J_{\alpha \beta} \equiv\left(\partial F_{\alpha} / \partial x^{\beta}\right)$ is regular everywhere, except at the limit point where it becomes singular. ${ }^{17}$ Thus to the set of nonlinear equations we add that

$$
\operatorname{det}\left[J_{\alpha \beta}\right]=0 \text {, }
$$

along with the addition of a new variable, say $E$. Then using $x_{w}$ as the homotopy parameter we can follow a homotopy of equilibrium solutions corresponding to the limit points on curves such as those in Figs. 1 and 3. The curve of Fig. 4 represents the curve of points where the stability of solutions changes. A plot of $x_{w}$ vs $T_{c}$ for these limit points looks very similar to Fig. 4, with $E_{c}$ replaced by $T_{w}$ and the plane is

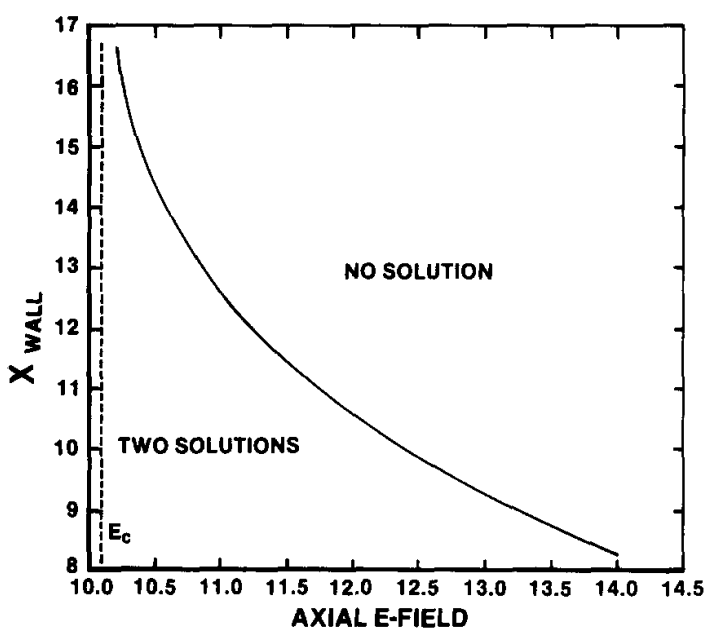

FIG. 4. A curve of $x_{w}$ vs $E$ at the limit points of the homotopy curves represented by Figs. 1 and 3. An equilibrium solution with values for $x_{w}$ and $E$ lying on this curve is marginally stable to thermal perturbations, i.e., the eigenvalue with smallest absolute magnitude equals zero and all other eigenvalues are negative.

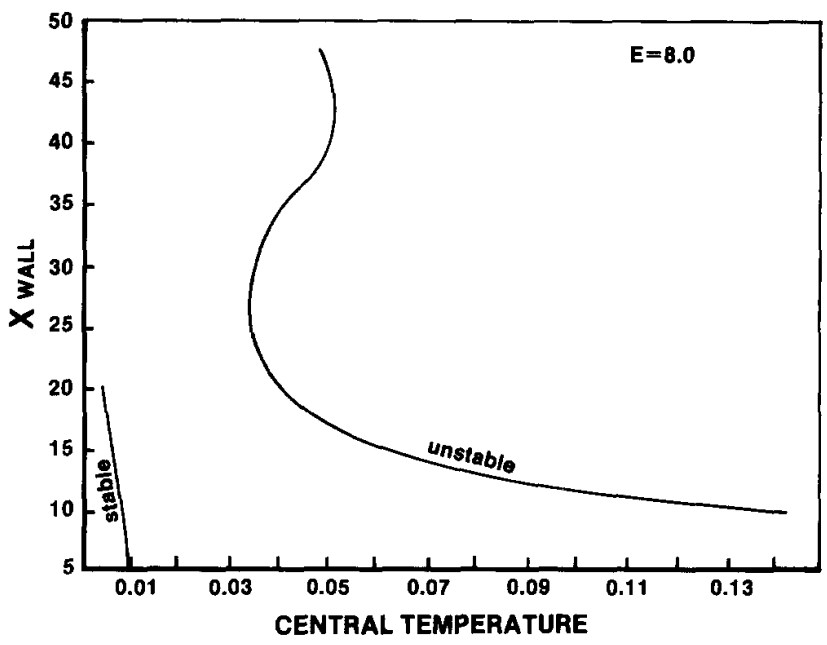

FIG. 5. Homotopy curves of $x_{w}$ vs $T_{c}$ for $E=8.0<E_{c}$. No longer are the stable and unstable branches joined through a limit point. Multiple unstable equilibria are possible for a given value of $T_{c}$.

divided in the same way into regions of solutions or no solutions.

For values of $E<E_{c}$ the stable and unstable branches of the equilibrium solutions are not connected. All of the stable solutions have a $T_{c}<T_{w}$ and the profiles have the temperature rising gradually from $T_{c}$ until they reach $T_{w}$ at $x_{w}$. The unstable solutions can exhibit oscillations of the profile about $T_{w}$ as $x \rightarrow x_{w}$. Figure 5 displays the solution homotopy curves for $E=8.0<E_{c}$. The multiple unstable solutions for a given $T_{c}$ are evident.

With this result it is clear that as $E$ decreases and approaches $E_{c}$, the $x_{w}$ vs $T_{c}$ curves becomes ever more sharply peaked near $T_{w}$ with $x_{w} \rightarrow \infty$. At $E=E_{c}$ the stable and unstable branches break and for $E<E_{c}$ the homotopy curves are qualitatively similar to those of Fig. 5 and exhibit multiple unstable equilibria.

In all situations previously discussed $B_{w}$ maintains a monotonic relationship to $T_{c}$. Curves of $B_{w}$ plotted against $E$ and $x_{w}$ are similar in character to those using $T_{c}$.

\section{B. Stability}

The stability of all equilibrium solutions was determined by computing the eigenvalues of the maxtrix $D$, symbolically written in Eq. (40). These eigenvalues turn out to be real and are, of course, only an approximation to a finite number of the eigenvalues in the spectrum of the partial differential operator in Eqs. (30) and (31). Table II exhibits the dependence of these eigenvalues on the number of finite elements chosen.

\section{DISCUSSION}

The foregoing analysis and results demonstrate that the LP method using finite elements and cubic $B$ splines gives an effective method for studying equilibrium solutions to problems in DMHD. When coupled with homotopy techniques, the dependence of solutions on parameters of the system can be extensively investigated and the stability studied. We note that these numerical techniques are not limited to certain functional forms for the dependence of transport coefficients 
TABLE II. Eigenvalues of the matrix $D$ of Eq. (40), tabulated with respect to the number of finite elements for an equilibrium with $x_{w}=4.0$ and $E=12.0$. For $N$ finite elements the dimension of $D$ is $2(N+1)$.

\begin{tabular}{|c|c|c|c|c|}
\hline \multicolumn{5}{|c|}{ Number of finite elements } \\
\hline $\begin{array}{c}5 \\
-62.78206 \\
-366.7265 \\
-368.0727 \\
-918.5126 \\
-1729.785 \\
-1865.642 \\
-2943.670 \\
-4563.562 \\
-4955.147 \\
-8554.193 \\
-14055.28 \\
-24361.69\end{array}$ & $\begin{array}{c}6 \\
-62.78199 \\
-366.7278 \\
-368.0683 \\
-917.8225 \\
-1716.758 \\
-1865.716 \\
-2799.082 \\
-4367.156 \\
-4561.537 \\
-6960.671 \\
-8476.029 \\
-13822.76 \\
-20609.37 \\
-34784.26\end{array}$ & $\begin{array}{c}7 \\
-62.78179 \\
-366.7285 \\
-368.0608 \\
-917.6970 \\
-1713.041 \\
-1865.755 \\
-2767.233 \\
-4136.517 \\
-4561.426 \\
-6057.741 \\
-8459.411 \\
-9337.676 \\
-13629.41 \\
-20405.29 \\
-28369.76 \\
-47179.43\end{array}$ & $\begin{array}{c}8 \\
-62.78195 \\
-366.7288 \\
-368.0688 \\
-917.6778 \\
-1712.059 \\
-1865.775 \\
-2755.825 \\
-4075.191 \\
-4561.542 \\
-5746.203 \\
-8006.846 \\
-8455.897 \\
-12086.87 \\
-13570.92 \\
-20053.65 \\
-28305.23 \\
-37327.84 \\
-61529.16\end{array}$ & $\begin{array}{c}9 \\
-62.78195 \\
-366.7290 \\
-368.0691 \\
-917.6795 \\
-1711.794 \\
-1865.787 \\
-2752.020 \\
-4049.529 \\
-4561.651 \\
-5645.728 \\
-7629.448 \\
-8453.235 \\
-10209.79 \\
-13553.89 \\
-15206.81 \\
-19914.36 \\
-27774.51 \\
-37516.55 \\
-47479.91 \\
-77819.09\end{array}$ \\
\hline
\end{tabular}

on the temperature and magnetic field. Furthermore, arbitrary source or sink terms depending on the field quantities can be included. The transport coefficients chosen here were chosen because of their familiarity. Anomalous forms for transport coefficients are easily studied in the same way but in the absence of any compelling motivation for one form over another we have not presented such results here.

For the system specifically studied there is a critical axial electric field $E_{c}$ for which there exist interesting temperature profiles peaked at the center, which are also stable to thermal fluctuations. There is in addition a maximum $E$ for which equilibrium solutions are possible. It is likely that before these bounds can be approached considerations of MHD stability become dominant. The question of dynamic equilibrium and stability has been completely ignored in this study and would be an appropriate extension. Future work will also include a variable $B_{z}$ field with dynamo terms.

\section{ACKNOWLEDGMENTS}

We have benefited from many useful discussions with our colleagues Grant Mason, Ross Spencer, and Phil Matheson. We would also like to acknowledge our debt to Gene Allgower for introducing us to the fascinations of homotopies and sharing with us models of codes.

This material is partially based upon work supported by the National Science Foundation under Grant No. INT8512193.

\section{APPENDIX: MATRIX DEFINITIONS}

$U_{i j}=\int_{0}^{N} \xi d \xi \frac{T_{0}^{2-n_{\mathrm{c}}}}{B_{0}^{2}} \phi_{i}^{\prime} \phi_{j}^{\prime}$

$V_{i}=\int_{0}^{N} \xi d \xi \phi_{i}\left[\frac{\mu}{T_{0}^{n_{r}}}\left(B_{0 \theta}^{\prime}+\frac{B_{0 \theta}}{\xi}\right)^{2}-\frac{\beta x_{w}^{2}}{N^{2}} T_{0}^{n_{B}}\right]$

$$
\begin{aligned}
& W_{\hat{j}}=\int_{0}^{N} \xi d \xi \psi_{\hat{i}}\left(\psi_{\hat{j}}^{\prime}+\frac{\psi_{\hat{j}}}{\xi}\right), \quad X_{\hat{i}}=\int_{0}^{N} \xi d \xi T_{0}^{n_{r}} \psi_{\hat{i}}, \\
& Y_{i j}=\int_{0}^{N} \xi d \xi \phi_{i} \phi_{j}, \quad Z_{i j}=\int_{0}^{N} \xi d \xi \tilde{\psi}_{i} \tilde{\psi}_{j}, \\
& A_{i j}=n_{c} \int_{0}^{N} \xi d \xi \frac{T_{0}^{\prime}}{B_{0}^{2} T_{0}^{n_{c}+1}} \phi_{i}^{\prime} \phi_{j}, \\
& B_{i j}=2 \int_{0}^{N} \xi d \xi \frac{B_{0 \theta} T_{0}^{\prime}}{\left(B_{0}^{2}\right)^{2} T_{0}^{n_{c}}} \phi_{i}^{\prime} \tilde{\psi}_{j} \text {, } \\
& C_{i j}=\int_{0}^{N} \xi d \xi \frac{1}{B_{0}^{2} T_{0}^{n_{c}}} \phi_{i}^{\prime} \phi_{j}^{\prime}, \quad D_{i j}=\int_{0}^{N} \xi d \xi T_{0}^{n_{B}-1} \phi_{i} \phi_{j}, \\
& E_{i j}=\left(\frac{x_{w} E}{N}\right)^{2} \int_{0}^{N} \xi d \xi T_{0}^{n_{r}-1} \phi_{i} \phi_{j}, \\
& F_{i j}=\frac{x_{w} E}{N} \int_{0}^{N} \xi d \xi \phi_{i}\left(\tilde{\psi}_{j}^{\prime}+\frac{\tilde{\psi}_{j}}{\xi}\right) \text {, } \\
& G_{i j}=\int_{0}^{N} \xi d \xi \frac{1}{T_{0}^{n_{r}}}\left(\tilde{\psi}_{i}^{\prime}+\frac{\tilde{\psi}_{i}}{\xi}\right)\left(\tilde{\psi}_{j}^{\prime}+\frac{\tilde{\psi}_{j}}{\xi}\right), \\
& H_{i j}=\int_{0}^{N} \xi d \xi \tilde{\psi}_{i}\left(\phi_{j} / T_{0}\right)^{\prime} \text {. }
\end{aligned}
$$

All integrals are performed using Gaussian four-point integration over each finite element of the interval.

'S. N. Rasband, G. W. Mason, and P. L. Matheson, submitted to J. Comput. Phys.

${ }^{2}$ P. Glansdorff and 1. Prigogine, Thermodynamic Theory of Structure, Stability and Fluctuations (Wiley, New York, 1971), Chap. X.

${ }^{3}$ I. Prigogine, in Nonequilibrium Thermodynamics, Variational Techniques, and Stability, edited by R. J. Donnelley, R. Herman, and I. Prigo- 
gine (University of Chicago, Chicago, 1966), p. 3

${ }^{4}$ D. F. Hayes, in Nonequilibrium Thermodynamics, Variational Techniques, and Stability, edited by R. J. Donnelly, R. Herman, and I. Prigogine (University of Chicago, Chicago, 1966), p. 17.

${ }^{5} \mathrm{P}$. Glansdorff, Physica 32, 1843 (1966).

${ }^{6}$ R. S. Schechter and D. M. Himmelblau, Phys. of Fluids 8, 1431 (1965).

${ }^{7}$ J. D. Legros and J. K. Platten, J. Non-Equilib. Thermodyn. 2, 211 (1977).

${ }^{8}$ S. M. Rohde, Physica 61, 53 (1972).

${ }^{9}$ S. M. Rohde, in Advances in Chemical Physics, edited by I. Prigogine and

S. A. Rice (Wiley, New York, 1975), Vol. 32, p. 37.

${ }^{10} \mathrm{~K}$. Georg, in Numerical Solution of Nonlinear Equations, edited by E. L. Allgower, K. Glashoff, and H. O. Peitgen (Springer, New York, 1980), Lecture Notes in Mathematics, Vol. 878, p. 128.
${ }^{11}$ K. Georg, SIAM J. Sci. Stat. Comput. 2, 35 (1981).

${ }^{12}$ E. L. Allgower and K. Georg, in Mathematical Programming: The State of the Art, edited by A. Bachem, M. Grötschel, and B. Korte (Springer, New York, 1982), p. 15.

${ }^{13}$ M. Braun, Differential Equations and Their Application (Springer, New York, 1983).

${ }^{14}$ D. Dobrott, R. L. Miller, and J. M. Rawls, Phys. Fluids 20, 1744 (1977). ${ }^{15}$ P. Rosenau, Phys. Fluids 25, 148 (1982).

${ }^{16} \mathrm{~J}$. Guckenheimer and P. Holmes, Nonlinear Oscillations, Dynamical Systems, and Bifurcations of Vector Fields (Springer, New York, 1983), Chap. 3.

${ }^{17}$ M. Kubicek and M. Marek, Computational Methods in Bifurcation Theory and Dissipative Structures (Springer, New York, 1983), Chap. 2. 